\title{
In Silico-guided Metabolic Engineering of Bacillus Subtilis for Efficient Biosynthesis of Purine Nucleosides by Blocking the Key Backflow Nodes
}

\section{Aihua Deng}

Institute of Microbiology Chinese Academy of Sciences

Qidi Qiu

Institute of Microbiology Chinese Academy of Sciences

Qinyun Sun

Institute of Microbiology Chinese Academy of Sciences

Zhenxiang Chen

Institute of Microbiology Chinese Academy of Sciences

Junyue Wang

Institute of Microbiology Chinese Academy of Sciences

Yu Zhang

Institute of Microbiology Chinese Academy of Sciences

Shuwen Liu

Institute of Microbiology Chinese Academy of Sciences

Tingyi Wen ( $\nabla$ wenty@im.ac.cn )

Institute of Microbiology Chinese Academy of Sciences https://orcid.org/0000-0003-2583-7088

\section{Research}

Keywords: Bacillus subtilis, genome-scale metabolic network model, metabolic engineering, back-flow node, purine nucleosides, chassis bacterium

Posted Date: November 29th, 2021

DOI: https://doi.org/10.21203/rs.3.rs-1091989/v1

License: (c) (i) This work is licensed under a Creative Commons Attribution 4.0 International License. Read Full License 


\section{Abstract}

Background: Purine nucleosides play essential roles in cellular physiological processes and have a wide range of applications in the fields of antitumor/antiviral drugs and food. However, microbial overproductions of purine nucleosides by de novo metabolic engineering have been a great challenge due to their strict and complex regulatory machinery involved in the biosynthetic pathways.

Results: In this study, we designed an in silico-guided strategy for overproducing purine nucleosides based on the genome-scale metabolic network model in Bacillus subtilis. The metabolic flux was analyzed to predict two key backflow nodes Drm (Purine nucleotides toward PPP) and YwjH (PPP-EMP) for resolving the competitive relationship between biomass and purine nucleotides synthesis. In terms of the purine synthesis pathway, the first backflow node Drm was inactivated to block the degradation of purine nucleotides and greatly increased the inosine production to $13.98-14.47 \mathrm{~g} / \mathrm{L}$ without affecting cell growth. Furthermore, releasing feedback inhibition of purine operon by promoter replacement further enhanced the accumulation of purine nucleotides. In terms of the central carbon metabolic pathways, deleting the second backflow node $\mathrm{YwjH}$ and overexpressing Zwf were combined to increase the inosine production to $22.01 \pm 1.18 \mathrm{~g} / \mathrm{L}$ by enhancing the metabolic flow of PPP. Through switching on the flux node of the glucose-6- phosphate to PPP or EMP, the final inosine engineered strain produced up to $25.81 \pm 1.23 \mathrm{~g} / \mathrm{L}$ of inosine by a pgi-based metabolic switch in shake-flask cultivation, suggesting the highest yield in de novo engineered inosine bacteria. Under the guidance of the in silico-designed strategy, a general chassis bacterium was generated for the first time to efficiently synthesize inosine, adenosine, guanosine, IMP, and GMP, providing the sufficient precursor for the synthesis of various purine intermediates.

Conclusions: Overall, in silico-guided metabolic engineering successfully optimized the purine synthesis pathway by exploring the efficient targets, representing a superior strategy for efficient biosynthesis of the biotechnological products.

\section{Background}

As the essential metabolites in the organisms, purine intermediates participate in the metabolic synthesis of nucleic acids, energy, and amino acids [1, 2]. Among them, purine nucleosides refer to adenosine, guanosine, inosine, and xanthosine that contain nucleosides and bases [3]. They have a wide range of applications in the fields of food and medicine as commercially important flavor enhancers and the precursor of antitumor/antiviral drugs [4, 5]. Especially nowadays, tumors and influenza viruses have seriously threatened the health of human beings, triggering increasing demands for nucleosides products in the whole world. Currently, microbial fermentation is the main approach for producing purine nucleosides, and investigations have been carried out to construct genetically engineered bacteria by metabolic engineering [4, 6-9]. The metabolism pathway shows that purine nucleosides are de novo synthesized via the Glycolysis pathway (EMP, Embden-Meyerhof-Parnas Pathway), Pentose Phosphate Pathway (PPP), and purine synthesis pathway (Additional file 1: Figure S1A). Firstly, glucose-6-phosphate, 
the intermediate product synthesized by the first step of EMP, is catalyzed by the 5-step enzymatic reactions of PPP to form phosphoribosyl pyrophosphate (PRPP). Then, PRPP is catalyzed to form inosine monophosphate (IMP) through 11 steps of enzymatic reactions in the purine synthesis pathway. Finally, the precursor IMP is catalyzed to form various purine nucleotides, nucleosides, and nucleobases through the terminal purine synthesis pathway. Meanwhile, nucleobases can be directly catalyzed by the phosphoribosyltransferases to form nucleotides in the salvage pathway (Additional file 1: Figure S1A). Thus, the synthesis of purine nucleosides requires a lot of precursors and energy, which is difficult to accumulate in the cell because of the strict and complex regulation $[3,10]$.

Bacillus is known to be superior for the fermentation of nucleosides with the advantages of a safe producer, few by-products, and a simple separation process $[11,12]$. In $B$. subtilis 168 , the inosine production was increased to $6 \mathrm{~g} / \mathrm{L}$ by disrupting the conversion of IMP to AMP and XMP, the degradation of inosine, and the repressor PurR and 5'-UTR of the pur operon [13]. Furthermore, the nucleoside efflux transporters have been reported to increase the production of extracellular purine nucleosides [9]. Under the guidance of transcriptional and metabolite pool analysis, overexpression of prs, purF, and purA in $B$. subtilis XGL have been reported to increase PRPP concentration and pur operon transcription, which achieved adenosine accumulation to $7.04 \mathrm{~g} / \mathrm{L}$ [7]. In 2019, Li et al inactivated the GMP synthetase gene gua $A$ and optimized the growth condition to increase adenosine production from 7.40 to $14.39 \mathrm{~g} / \mathrm{L}$ in $B$. subtilis A509 [6]. Besides, Escherichia coli is another industrial strain that is applied to nucleoside synthesis, which has been reported to produce $6.7-7.5 \mathrm{~g} / \mathrm{L}$ inosine by overexpressing the prs and purF genes and inactivating the purA, deoD, purF, purR, add, edd, pgi, and xapA genes [14]. In our previous study, a de novo engineered strain with a clear genetic background was constructed to accumulate 7.6 $\mathrm{g} / \mathrm{L}$ inosine by deleting the purA and deoD genes in B. subtilis W168 [12]. The flux distribution of the whole-cell revealed that purine synthesis has a long metabolic pathway and its carbon flux is carried by PPP, which is not the mainly intracellular carbon metabolism. Furthermore, nucleosides synthesis is strictly and complexly regulated in the cell, remaining a grand challenge for overproduction of nucleosides by de novo metabolic engineering $[15,16]$. Overall, previous efforts to construct engineered strains mostly focus on the metabolic pathways directly associated with the purine nucleosides biosynthesis, which often resulted in slow growth and relatively low production of desired metabolites $[12,17]$. Therefore, it is urgently required to adopt a rational-designed engineering strategy to achieve the effective synthesis of purine nucleoside.

With the rapid development of whole-genome sequencing and multi-omics technologies, the genomescale metabolic network models (GEM) have been developed by constructing the metabolic network with aid of the computer technology $[18,19]$. Based on the principle of gene-protein-reaction relationships, GEM is constructed to link the genes to the proteins that catalyzes reactions in the network. A series of biochemical reactions in the cell are responsible for the synthesis of energy and metabolites. Thus, the models can be widely used in quantitative predictions of cell growth and desired product under metabolically/environmentally disturbed conditions [20]. Until now, GEM have been undergone multiple generations of upgrades to continuously improve the completeness of models, the accuracy of prediction, and the scope of application in industrial microorganisms, such as E. coli, B. subtilis, Corynebacterium 
glutamicum, and Saccharomyces cerevisiae [19, 21-23]. Among them, the BBsu1103V2 model of $B$. subtilis accounts for 1103 open reading frames and 1451 metabolic reactions involving 1156 metabolites. Compared with the available models in $B$. subtilis, it has been improved considerably at predictions of metabolites and viability of mutant strains in rich defined medium and glucose minimal media. Thus, the model provides an effective tool to conduct rational guidance for the metabolic engineering and systems biology research in B. subtilis [24].

In this study, we used the IBsu1103V2 model to analyze and predict the novel targets for increasing IMP flux without influencing biomass $[25,26]$. An in silico-guided engineering strategy was designed to disrupt the backflow node (the pentose phosphate mutase Drm) of Purine synthesis pathway towards PPP, release feedback inhibition of the purine operon, block the backflow node (the transaldolase YwjH) of PPP to EMP, and regulate the key enzymes' expressions. Under the guidance of the rational-designed strategy, a universal chassis strain was de novo constructed to accumulate various purine intermediates. The final engineered strain showed the highest yield of inosine in de novo engineered bacteria, being of great significance for further understanding of purine metabolites biosynthesis and its regulatory mechanism.

\section{Methods}

\section{Strains, medium, and chemicals}

The strains used in this study were listed in Additional file 1: Table S1. EC135 lacking all R-M systems and orphan MTases was used as a cloning host to construct plasmids [39]. All strains were cultured in Luria-Bertani (LB) medium (10 g/L tryptone, $5 \mathrm{~g} / \mathrm{L}$ yeast extract, and $10 \mathrm{~g} / \mathrm{L} \mathrm{NaCl}$ ) supplemented with the appropriate antibiotics $(100 \mu \mathrm{g} / \mathrm{mL}$ ampicillin were used in E. coli and $20 \mu \mathrm{g} / \mathrm{mL}$ erythromycin, $10 \mu \mathrm{g} / \mathrm{mL}$ kanamycin, and $10 \mu \mathrm{g} / \mathrm{mL}$ chloromycetin were used in B. subtilis).

Kits for DNA purification/gel recovery and extracting genome DNA, plasmid DNA, and RNA were purchased from the TIANGEN Biotech (Beijing, China). DNA polymerase, restriction enzymes, and dNTP were purchased from New England Biolabs (USA). Antibiotics, inducers, and standard chemicals, such as ampicillin, kanamycin, chloromycetin, erythromycin, IPTG, xylose, purine bases, nucleotides, and nucleosides were purchased from the Sigma-Aldrich (USA). Tryptone and Yeast Extract were purchased from Oxoid Company (UK). Other reagents for cell culture and fermentation medium were all analytical pure, purchased from Beijing Modern Oriental Fine Chemical Co., Ltd (China).

\section{DNA manipulation for plasmid and strain construction}

The TCCRAS method was used for scarless manipulating the bacterial genomes as described by our previous studies[40]. To construct the recombinant plasmids for gene modifications in $B$. subtilis, the upstream and downstream DNA fragments flanking the targets purA, pupG, deoD, drm, $P_{\text {pun }} y w j H$, pgi, and $z w f$ were amplified using the W168 genome DNA as the template. The purified DNA fragments were jointed by Splice Overlap Extension (SOE) PCR and then ligated with the vector pWYE486 using the 
digestion-ligation approach or NEBuilder HiFi DNA fragment rapid assembly kit (New England Biolabs, USA). After verification by PCR and DNA sequencing, the recombinant plasmids were separately transformed into the competent cells of $B$. subtilis to generate the engineering strains through two rounds of homologous recombination (Additional file 1: Table S1). The detailed procedures for constructing recombinant plasmids, preparing competent cells, and screening engineered strains were carried out according to the previous method [40,41]. All of the primers used in this study were shown in Additional file 1: Table S2 and synthesized by Invitrogen (Shanghai, China). PCR amplification was performed with Q5 DNA polymerase (New England Biolabs, USA) following the procedure of the manufacturer. DNA sequencing was performed by Beijing Genomics Institute (BGI, Beijing, China) and Tianyi Huiyuan Biotechnology Co., Ltd (China).

\section{Constraint-based metabolic flux analysis}

In silico analysis of metabolic flux for IMP production was performed by the iBsu1103V2 model [24]. The main features of this model were shown in Additional file 1: Table S3. During the simulation calculation, the medium used in the $B$. subtilis model is the minimum medium. The upper and lower limits of the flux for each exchange reaction in the model are set as the previous method [42]. (i) $\mathrm{H}_{2} \mathrm{O}, \mathrm{O}_{2}, \mathrm{CO}_{2}, \mathrm{Ca}^{2+}, \mathrm{H}^{+}, \mathrm{K}^{+}$, $\mathrm{Mg}^{2+}, \mathrm{Na}^{+}, \mathrm{Fe}^{3+}$, and other substances can freely enter or exit the metabolic network for exchange. Thus, the upper limit of the reaction flux was set to $1000 \mathrm{mmol}^{\circ} \mathrm{gDW}^{-1} \cdot \mathrm{h}^{-1}$ (millimoles per gram of dry weight per hour), and the lower limit was set to $-1000 \mathrm{mmol} \cdot \mathrm{g} \mathrm{DW}^{-1} \cdot \mathrm{h}^{-1}$. (ii) When Glucose was set as a carbon source, the maximum uptake rate was set as $1000 \mathrm{mmol} \cdot \mathrm{gDW}^{-1} \cdot \mathrm{h}^{-1}$ and the minimum rate was set to -1.8 $\mathrm{mmol} \cdot \mathrm{g} \mathrm{DW}^{-1} \cdot \mathrm{h}^{-1}$. For the absorption rate of nitrogen source $\mathrm{NH}_{3}$, phosphorus source $\mathrm{Pi}$, and sulfur source Sulfate, the upper limit was set as $1000 \mathrm{mmol} \cdot \mathrm{gDW}^{-1} \cdot \mathrm{h}^{-1}$ and the lower limit was set as $-5 \mathrm{mmol} \cdot \mathrm{g} \mathrm{DW}^{-1} \cdot \mathrm{h}^{-}$ 1 (Fig. 2a).

Flux Balance Analysis (FBA) was used for analyzing the metabolic network of $B$. subtilis. The organism was assumed in a pseudo-steady state that was the concentration of each intermediate metabolite was unchanged [43]. The calculation principle of FBA could be expressed by the following formula:

Maximize: $\mathrm{C}^{\top} \cdot \mathrm{v}$

Subject to: $S \cdot v=0$

$$
\mathrm{v}_{\min } \leq \mathrm{v} \leq \mathrm{v}_{\max }
$$

Where $\mathrm{C}_{\mathrm{T}}$ is the objective function value, expressed as a function of the flux of each reaction; $\mathrm{S}$ is the $\mathrm{m} \times \mathrm{n}$ order stoichiometric matrix; $v$ is the reaction flux; $v_{\max }$ is the upper limit flux of each reaction; $v_{\min }$ is the lower limit flux of each reaction. In general, the accumulation rate of biomass was used as an objective function to calculate the metabolic flux distribution in the case of maximizing biomass. 
Genetic design through local search (GDLS) converts the bi-level optimization problem (metabolic flow of biomass and IMP in this study) into a mixed-integer linear programming problem. An effective, lowcomplexity multi-path search was carried out to find the transformation target in the solution space that maximizes the target metabolic flow under the precursor of a certain growth rate of the strain. The initial calculation principle was designed as the previous method [25]

The regulatory on/off minimization (ROOM) was determined by the smallest number of reactions, which could change the metabolic flow of the modified strain [44]. These reactions would be used as objective functions for the analysis of flow disturbance after metabolic engineering. The calculation principle is as follows:

$$
\operatorname{Min} \sum_{i=1}^{m} y_{i}
$$

$$
\begin{aligned}
& S \cdot v=0 \\
& v_{\min } \leq v \leq v_{\max } \\
& v_{j}=0, j \in A \\
& \quad \text { for } 1 \leq i \leq m \\
& v_{i}-y_{i}\left(v_{\max , i}-w_{i}^{u}\right) \leq w_{i}^{u} \\
& v_{i}-y_{i}\left(v_{\min , i}-w_{i}^{b}\right) \geq w_{i}^{l} \\
& y_{i} \in\{0,1\} \\
& w_{i}^{u}=w_{i}+\delta\left|w_{i}\right|+\varepsilon \\
& w_{i}^{b}=w_{i}+\delta\left|w_{i}\right|+\varepsilon
\end{aligned}
$$

Among them, $\operatorname{Min} \sum_{i=1}^{m} \mathrm{yi}^{\prime}$ is the value of the objective function, which is expressed as the total number of reactions that change the metabolic flux. $S$ is the stoichiometric $m \times n$ matrix, $v$ represents the reaction flux vector, $v_{\max }$ represents the upper limit of each reaction flux, $v_{\min }$ represents the lower limit of each reaction flux, and $w$ represents the reaction flux vector of the wild-type strain. The upper threshold is specified by $w_{i}{ }^{u}$ and the lower threshold is specified by $w_{i}{ }^{1}$.

\section{Shake-flask cultivation}

To determine the production of purine nucleotide/base/nucleoside metabolites, the strains were precultured in seed medium ( $20 \mathrm{~g} / \mathrm{L}$ glucose, $10 \mathrm{~g} / \mathrm{L}$ sodium glutamate, $20 \mathrm{~g} / \mathrm{L}$ peptone, $20 \mathrm{~g} / \mathrm{L}$ yeast powder, $5 \mathrm{~g} / \mathrm{L}$ corn steep liquor, $2.5 \mathrm{~g} / \mathrm{L} \mathrm{NaCl}, 1 \mathrm{~g} / \mathrm{L}$ urea, $\mathrm{pH} 7.2)$ at $32^{\circ} \mathrm{C}$ and $220 \mathrm{rpm}$. Until the $\mathrm{OD}_{600}$ reached 10-12, three milliliters of seed culture was inoculated into a $500-\mathrm{mL}$ shake flask containing 30 
$\mathrm{mL}$ of fermentation medium ( $120 \mathrm{~g} / \mathrm{L}$ glucose, $16 \mathrm{~g} / \mathrm{L}$ soybean meal hydrolysate, $14 \mathrm{~g} / \mathrm{L}$ yeast powder, 15 $\mathrm{g} / \mathrm{L}\left(\mathrm{NH}_{4}\right)_{2} \mathrm{SO}_{4}, 4 \mathrm{~g} / \mathrm{L} \mathrm{MgSO}_{4} \cdot 7 \mathrm{H}_{2} \mathrm{O}, 4 \mathrm{~g} / \mathrm{L} \mathrm{K}_{2} \mathrm{HPO}_{4}, 0.01 \mathrm{~g} / \mathrm{L} \mathrm{FeSO}_{4}, 0.1 \mathrm{~g} / \mathrm{L}$ biotin, and $20 \mathrm{~g} / \mathrm{L} \mathrm{CaCO}, \mathrm{pH}$ 7.0 ). According to our previous method [12], the cells were grown at $36^{\circ} \mathrm{C}$ and $220 \mathrm{rpm}$, and the medium $\mathrm{pH}$ was adjusted to 7.0 by supplementation with ammonia. At least three independent experiments were repeated to show the average data, standard deviations, and statistical significances.

\section{Quantitative real-time PCR}

The bacterial total RNA was extracted by the RNA isolation kit and NanoDrop 2000c (Thermo Fisher, USA) was used to determine the concentration of RNA sample, which was then subjected to cDNA synthesis using a FastQuant RT Kit. The primers for qRT-PCR were listed in Supplementary Table S2. The cDNA was used as the template for the qRT-PCR experiment using a LightCycler® 96 Real-Time PCR System (Roche, Switzerland). The 16S rRNA gene was used as the reference gene to normalize the mRNA levels of target genes. The negative controls were designed in each PCR reaction to exclude DNA and other contaminants. A melting curve analysis and the Rotor-Gene Q series software (Qiagen, Germany) with the $2^{-\Delta \Delta C T}$ method were used to verify and analyze qPCR data[45]. The qRT-PCR method was used to detect the transcription levels of target genes. At least three repeated experiments for each sample were carried out.

\section{Analytical methods}

Cell density was measured by determining the absorbance at $600 \mathrm{~nm}\left(\mathrm{OD}_{600}\right)$ using a spectrophotometer (V-1100D; Mapada Instruments, Shanghai, China). After dilution and filtration of the culture supernatant and cell extract with $\mathrm{ddH}_{2} \mathrm{O}$, nucleoside metabolites were determined using a High-Performance Liquid Chromatography (HPLC) system (Agilent, USA) with an SB-AQ column $(4.6 \times 250 \mathrm{~mm}, 5 \mu \mathrm{m}$, Agilent) at $33^{\circ} \mathrm{C}$. Mobile phase $A$ was $100 \%(\mathrm{v} / \mathrm{v})$ methanol, whereas mobile phase $B$ consisted of $0.5 \%(\mathrm{~W} / \mathrm{V})$ $\mathrm{KH}_{2} \mathrm{PO}_{4}$ at a pH of 4.5. The HPLC was performed using a ratio of $90 \% \mathrm{~A}$ and $10 \% \mathrm{~B}$ at $1 \mathrm{ml} / \mathrm{min}$ with a monitor at $360 \mathrm{~nm}$. All measurements were performed at least in triplicate and standard deviations (SD) were calculated from three independent experiments.

\section{Results}

\section{Initial biosynthesis of purine nucleosides by the traditional engineering method}

As shown in the purine synthesis pathway of $B$. subtilis, IMP is the important precursor for synthesizing various purine nucleosides by 1-3 steps of catalytic reactions. Among various purine nucleosides, inosine is directly synthesized from IMP through one-step reaction (Fig. 1a). To rapidly evaluate the engineering effect, the inosine production was used as an indicator to determine the metabolic flow of purine nucleosides in this study (Fig. 1b). Thus, the purA gene encoding adenylosuccinate synthetase was first knocked out to block the adenosine synthesis branch and increase the inosine accumulation of the engineered strain PN01 (W168 $\triangle p u r A$ ). Then, the purine nucleoside phosphorylases (PNP) DeoD and/or 
PupG that involved in the degradation of nucleosides were inactivated to generate the engineered strains PN02 (W168 $\Delta p u r A \Delta d e o D)$, PN03 (W168 $\Delta p u r A \Delta p u p G)$, and PN04 (W168 $\Delta p u r A \Delta p u p G \Delta d e o D)$.

After shake-flask cultivation of $72 \mathrm{~h}$, the strain PN01 could accumulate $0.62 \pm 0.16 \mathrm{~g} / \mathrm{L}$ of inosine and $4.63 \pm 0.22 \mathrm{~g} / \mathrm{L}$ of hypoxanthine formed by the degradation of inosine. The inosine production increased in the pupG-or/and deoD-deficient strains PN02 (2.21 $\pm 0.88 \mathrm{~g} / \mathrm{L})$, PN03 $(8.88 \pm 1.10 \mathrm{~g} / \mathrm{L})$, and PN04 $(9.54 \pm 1.19 \mathrm{~g} / \mathrm{L})$, while the degradation of inosine (as shown by the hypoxanthine production) continuously decreased in these strains. The results suggested that purA inactivation produced a high concentration of hypoxanthine by inosine degradation, which was significantly reduced by deleting the pupG and deoD genes. However, cell growths of the strains PN02, PN03, and PN04 were greatly decreased, especially slow for PNO3 and PN04 in seed cultivation, which were negatively affects the engineered strain's characteristics and not ideal for further metabolic engineering (Fig. 1c and d). Since the synthesis of nucleosides is strictly and complicatedly regulated in the cell, it is difficult to achieve the proper balance between cell growth and nucleoside production using traditional metabolic engineering. Therefore, it is necessary to adopt a rational-designed engineering strategy to search for optimal targets to resolve the problem.

\section{In silico prediction of the novel targets for maximizing synthesis of IMP}

Because IMP is the essential precursor of all purine nucleosides, the relationships between nucleosides flux and biomass as well as IMP productivity were investigated by constraint-based metabolic flux balance analysis based on the IBsu1103V2 model (Additional file 1: Table S3). Through flux balance analysis (FBA), the maximum theoretical growth rate of cells, the synthetic flux of IMP, and the conversion rate of IMP were $0.26 \mathrm{~h}^{-1}, 1.52 \mathrm{mmol}^{\circ} \mathrm{gDW}^{-1} \cdot \mathrm{h}^{-1}$, and $0.84 \mathrm{~mol} / \mathrm{mol}$ (IMP/glucose), respectively (Fig. 2a). Robustness analysis was further used to analyze the interaction relationship between the metabolic flux of IMP synthesis and cell biomass. The results showed that the production rate of IMP decreased with the continuous increase of cell growth rate, indicating a competitive relationship between the biomass and IMP synthesis. The highest IMP production would result in biomass being zero (Fig. 2b). Thus, a rationally engineering strategy was urgently required to balance flux distribution between biomass and IMP production.

The genetic design through local search (GDLS) was used to analyze and predict the optimal targets (Fig. 2c). The reaction formulas catalyzed by the enzymes from EMP, PPP and the purine synthesis pathway were selected as the potential targets to stimulate IMP production. Two strategies were obtained to strengthen the metabolic flow of IMP. Strategy 1 was designed to knock out the pentose phosphate mutase encoded by the $d r m$ gene and the transaldolase encoded by the $y w j H$ gene. Strategy 2 was designed to knock out the $d r m$ and the fructose bisphosphate aldehyde carboxylase encoded by $f b a A$ (Additional file 1: Table S4).

Since the predicted targets catalyze other reactions at the same time, whether they can be used as the ideal targets for enhancing IMP flux was further analyzed by the regulatory on/off minimization 
(Additional file 1: Table S5). Firstly, the upper and lower lines of the reactions catalyzed by the predicted targets were set to be "0" using the ChangeRxnBounds command (Fig. 2d). Subsequently, the minimum switch adjustment algorithm (ROOM) was used to access the effects of two strategies on biomass formation and IMP production by the MATLAB software. The results showed that Scheme 2 could remarkably increase the IMP flux by 3.47 folds (from 0.07 to $0.31 \mathrm{mmol}^{\circ} \mathrm{gDW}^{-1} \cdot \mathrm{h}^{-1}$ ), but the growth rate of mutant strain drops to zero. Scheme 1 could increase the IMP flux by $18 \%$ (from 0.07 to $0.08 \mathrm{~h}^{-1}$ ). More importantly, the growth rate of the mutant strain would only reduce to be 0.24 , a slightly decrease of $8 \%$ compared to the wild-type strain (Fig. 2d). As shown in the metabolic pathway, the predicted targets Drm and $\mathrm{YwjH}$ of scheme 1 are the key backflow nodes of the purine nucleotides to PPP and PPP to EMP, respectively (Fig. 3a). Therefore, the first backflow node Drm and the purine operon were selected as a new combination to rationally optimize the purine synthesis pathway. Furthermore, the second backflow node YwjH and the glucose 6-phosphate dehydrogenase Zwf were combined to increase the metabolic flow of PPP, which could supply more carbon flux for the synthesis of purine nucleotides.

\section{Blocking the degradation of purine nucleosides into PPP by inactivating the backflow node Drm}

To validate the effect of in silico-predicted target on the degradation of purine nucleosides, the gene $\mathrm{drm}$ was genetically modified by promoter knockout, nonsense mutation, and ORF (opening reading frame) deletion to construct the engineered strains PN05 (PN01 $\left.\Delta P_{d r m}\right)$, PN06 (PN01 drm*), and PN07 (PN01 $\Delta d r m)$ (Fig. 3B). Since the $d r m$ and $p u p G$ genes are located in the same operon, the three mutations might have different effects on the pupG expression, which were subsequently detected by the real-time quantitative reverse transcription PCR (qRT-PCR). The mRNA expression levels of the pupG gene of PN06 and PN07 strains were up-regulated 2-7 folds compared to the original strain PN01, but it could not be detected in PN05 due to the promoter deficiency (Fig. 3c). Compared with the nonsense mutation, deleting the $d r m$ gene considerably increased the expression of the pupG gene due to the shortened distance between the promoter and ORF.

After shake-flask cultivation, the growth of PN05, PN06, and PN07 strains were marginally lower than the original strain PN01, while similar growths were observed in the seed culture (Fig. 3d). The results indicated that the Drm inactivation would not significantly affect cell growth. The inosine productions of PN05, PN06, and PN07 strains reached 13.98-14.47 g/L, notably increasing above 20 folds compared to the original strain PN01 (Fig. 3e). Interestingly, Drm inactivation in different ways all remarkably reduced the hypoxanthine synthesis from $4.61 \mathrm{~g} / \mathrm{L}$ to $0.21 \mathrm{~g} / \mathrm{L}$.

To clarify the effects of Drm and PupG on the degradation of purine nucleosides, complementary experiments were carried out by separately expressing the drm and pupG genes in the PN05 (Additional file 1: Fig. S1B). The pupG expression strain PN05-s2 (PN05 lacA:: $P_{x y l}-p u p G-x y / R$ ) produced high inosine and low hypoxanthine, which were similar to the control strains PN05 and PN05-s0 (PN05 lacA:: $P_{x y l}{ }^{-}$ $x y / R)$. In the contrast, dramatically decreased inosine and increased hypoxanthine productions were detected in the $d r m$ expression strain PN-5-s1 (PN05 lacA:: $\left.P_{x y l}-d r m-x y / R\right)$. Combining above results, Drm was proved to exhibit a major effect on increasing inosine production and decreasing its degradation, not 
the purine nucleoside phosphorylase PupG (Fig. 3e and Additional file 1: Figure S1B). It can be inferred that Drm is a key backflow node for blocking the degradation of purine nucleosides into PPP and promoting inosine accumulation. Therefore, the in silico-predicted target can effectively improve the biosynthesis of purine nucleosides without obvious impact on the cell growth, which is superior to the traditional engineering targets (Fig. 1).

\section{Releasing complex regulation of purine operon to increase the synthesis of purine intermediates}

The purine operon is strictly regulated by the transcription initiation repression and ribosome-mediated switch in the cell (Fig. 4a). Optimization of the purine operon by promoter replacement was used to increase the purine nucleoside synthesis. After analysis by DNAMAN and RBS calculator v1.1 [27], different strengths of promoters $\left(\mathrm{P}_{43}, \mathrm{P}_{\text {veg, }} \mathrm{P}_{c t o}\right.$ and $\left.\mathrm{P}_{g s i B}\right)$ with different secondary structures were selected to replace the promoter $\mathrm{P}_{\text {pur }}$ of the purine operon. Among them, $\mathrm{P}_{c t c}$ formed the least stem-loop structure with the highest translation initiation efficiency of $35619.97 \mathrm{AU}$. The stem-loop structure of $\mathrm{P}_{\text {veg }}$ is far from the ribosome binding site (RBS) and the start codon with the initiation efficiency of 19842.93 $\mathrm{AU}$. The promoters $\mathrm{P}_{43}$ and $\mathrm{P}_{g s i B}$ closing to RBS and the initiation codon, formed a relatively complex stem-loop structure, which might result in a negative impact on translation initiation. $P_{43}$ forming the most stable stem-loop structure had the lowest translation initiation efficiency of $1274.25 \mathrm{AU}$, whereas $\mathrm{P}_{\text {gsiB }}$ had the translation initiation efficiency of $15147.28 \mathrm{AU}$.

To detect the effects of different promoters on the transcription levels of purine operon and the synthesis of purine intermediates, the promoter $\mathrm{P}_{\text {pur }}$ in wild-type strain W168 was separately replaced by $\mathrm{P}_{43}, \mathrm{P}_{\text {veg }}$, $\mathrm{P}_{\text {cto }}$ and $\mathrm{P}_{\text {gsiB }}$. The mRNA levels of purE and purF genes were all up-regulated 8-78 folds under control of the replaced promoters, suggesting that the transcription levels of purine operon were enhanced by the promoter replacement (Fig. 4b). After flask cultivation, purine intermediates of inosine, guanosine, and hypoxanthine were increased in the engineered strains (Fig. 4c). The promoter $\mathrm{P}_{\text {veg }}$ produced the highest accumulation of $1.24 \pm 0.10 \mathrm{~g} / \mathrm{L}$ purine intermediates, a 4.93 -fold improvement in comparison with the W168 strain $(0.25 \pm 0.07 \mathrm{~g} / \mathrm{L})$. The results indicated that $P_{\text {veg }}$ could efficiently relieve the complex regulation of purine operon and dramatically enhance the synthesis of the purine nucleotides, which was selected to optimize the inosine engineered strains.

To demonstrate the effect of promotor replacement on the inosine production, $\mathrm{P}_{\text {pur }}$ in the engineered strains was replaced by $\mathrm{P}_{\text {veg }}$ to generate PN09 (W168 $\left.P_{\text {pur }}: P_{\text {veg }}\right)$, PN12 (PN01 $\left.P_{\text {pur }}: P_{\text {veg }}\right)$, PN13 (PN04 $\left.P_{\text {pur: }}: P_{\text {veg }}\right)$, and PN14 (PN07 $\left.P_{\text {pur }}: P_{\text {veg }}\right)$. Flask cultivation showed that inosine productions of PN08 and PN13 did not significantly increase compared to their original strains W168 and PN04 (W168 $\triangle p u r A \Delta p u p G$ ) (Fig. 4d). However, the accumulation of hypoxanthine increased by 1-35 folds in these strains, demonstrating that the enhanced inosine was degraded (Additional file 1: Figure S2). When $P_{\text {pur }}$ in PN01 (W168 $\Delta$ purA) and PN07 (W168 $\Delta$ purA $\Delta d r m$ ) strains were replaced with $P_{\text {veg, }}$, the inosine production of PN12 and PN14 remarkably increased by $148 \%$ and $21 \%$, respectively. The accumulations of hypoxanthine in PN12 and PN14 were similar to their original strains (Additional file 1: Figure S2). 
Further improvement in the in silico-designed strain PN07 significantly increased the inosine production to $16.86 \pm 0.78 \mathrm{~g} / \mathrm{L}$, but the same modification was not effective in the traditionally engineered strain PN04 (Fig. 4d).

\section{Enhancing the PPP flow by blocking the backflow node YwjH and overexpressing the key enzyme Zwf}

Based on the guidance of GEM, there is a complex exchange of metabolic flux between EMP and PPP. To supply more carbon flux for the purine synthesis, the flow of PPP to EMP could be weakened by inactivating the backflow node YwjH. Meanwhile, the flow of Glucose to PPP could be strengthened by regulating the key enzymes' expression (Fig. 5a). Based on this, the Glucose-6-phosphate would be effectively catalyzed by the glucose 6-phosphate dehydrogenase encoded by the zwf gene to enter into PPP, and thereof supply more carbon flux for the synthesis of purine nucleotides. According to in silico design, the gene $y w j H$ was knocked out to generate the engineered strain PN15 (PN14 $\Delta y w j H)$. The inosine production of PN15 was significantly increased to $20.89 \pm 0.69 \mathrm{~g} / \mathrm{L}$ without apparent reduction of cell growth (Fig. 5A and Additional file 1: Figure S3). It indicated that $\mathrm{YwjH}$ inactivation could significantly promote nucleoside biosynthesis in accordance with in silico prediction. To further strengthen the metabolic flow of PPP, the $z w f$ gene controlled by a strong promoter was integrated into the genome to generate the strain PN16 (PN15:: $\mathrm{P}_{43}-z W f$ ), which increased the inosine production to $22.01 \pm 1.18 \mathrm{~g} / \mathrm{L}$ (Fig. 5a). Therefore, the synthesis of purine nucleosides was considerably improved by blocking the essential backflow node and overexpressing the key enzyme Zwf to enhance the PPP flow.

\section{Efficient production of inosine by dynamically switching metabolic flux of biomass and product}

To coordinate biomass and desired metabolites, a dynamic switch was constructed to allocate the metabolic flux into EMP and PPP. Two regulation strategies were designed to dynamically control the flow of glucose 6-phosphate into EMP or PPP. In the flow node of glucose to PPP, the scaffold proteins GBD, SH3, and PDZ were separately used to linearly assemble three key enzymes encoding by $g / c K, z w f$, and $y k g B$ genes (Fig. 5b). In the presence of IPTG, the engineered strain PN18 (PN16/pHT01-scaf-glcK$z w f-y k g B$ ) showed a similar inosine production, but a slight reduction of cell growth in comparison with the control strain PN17 (PN16/pHT01-scaf) (Additional file 1: Figure S4). In the absence of an inducer, the inosine production was increased to $22.33 \pm 0.65 \mathrm{~g} / \mathrm{L}$ without an obvious impact on cell growth. Thus, the cell growth and inosine production did not change significantly by the addition of the inducer, suggesting that the linear enzymes system was not ideal as a switch.

To dynamically control the metabolic flow of glucose into EMP, the pgi gene was knocked out or conditionally expressed by the inducible promoter $\mathrm{P}_{x y l}$ (Fig. 5c). The flask cultivation of the engineered strains PN19 (PN16 $\Delta p g i)$ and PN20 (PN16 $\left.P_{p g i}: P_{x y l}\right)$ showed that pgi deficiency inhibited cell growth and reduced the inosine synthesis (Fig. 5c and Additional file 1: Figure S5). While the pgigene was conditionally controlled by $\mathrm{P}_{x y /}$, the cell growth in the seed medium was improved as the inducer concentration increased from 0 to $3 \%$ (Additional file 1: Figure S6). Remarkably, the inosine production of strain PN20 reached $22.81 \pm 0.82 \mathrm{~g} / \mathrm{L}$ in the absence of inducer but dropped to $16.47 \pm 0.04 \mathrm{~g} / \mathrm{L}$ by 
overexpressing pgi in the presence of xylose (Fig. 5c). Thus, weakening pgi expression in the absence of an inducer was more effective to enhance the inosine production than the overexpression and deletion. It might be that the low expression level of $p g i$ weakens the central carbon metabolism, which could divert metabolic flux to inosine synthesis (Fig. $5 \mathrm{c}$ and Additional file 1: Figure S5). On the contrary, overexpression of pgiby the addition of an inducer could enhance the central carbon metabolism, which would promote cell growth and thereby decrease inosine synthesis (Fig. 5C and Additional file 1: Figures S5, S6). Therefore, the $P_{x y}$ driven pgi could be used as an effective metabolic switch to control cell growth and inosine biosynthesis, which was suitable to dynamically allocate the metabolic flow of PPP and EMP.

Based on the above results, a two-stage fermentation based on the metabolic switch was further adapted to improve inosine production. In the cell growth stage, xylose was added to increase the pgi expression, and thereof enhance the EMP flow to promote biomass. In the inosine production stage, the $p g i$ expression was reduced to weaken the EMP flow by removing the inducer, resulting in the flow enhancement of the PPP and purine nucleosides (Fig. 5d). Under the optimized fermentation process, the final engineered PN20 produced up to $25.81 \pm 1.23 \mathrm{~g} / \mathrm{L}$ of inosine with a yield of $0.32 \mathrm{~mol} / \mathrm{mol}$ (Inosine/glucose) in the shake-flask fermentation. Therefore, the metabolic switch was successfully developed to dynamically regulate the metabolic flow and maximizing the inosine production.

\section{Construction of the universal purine chassis strain}

Under the guidance of in silico design by GEM, the synthesis of inosine was remarkably improved by blocking the back-flow nodes, releasing feedback inhibition of the purine operon, and regulating the expression of key enzymes (Fig. 6a). Based on the in silico-guided engineering strategy, a general chassis bacterium for efficiently synthesis of various purine intermediates was constructed

by expressing the purA gene in the finally optimized strain. The purine metabolites of the universal chassis strain were detected by HPLC after flask cultivation. The detecting condition of each standard was optimized to separate each purine base, nucleotide, or nucleoside well (Fig. 6b). Purine intermediates of AMP, IMP, GMP, adenine, hypoxanthine, guanine, inosine, and guanosine were detected in the universal chassis strain (Fig. 6c). Among these metabolites, the accumulations of IMP, GMP, inosine, and guanosine were remarkably increased in PN17, whereas AMP and adenosine were reduced. As a decomposition product of inosine, the hypoxanthine concentration was extremely low. The accumulation level of purine intermediates was ranked as $\mathrm{IMP}>$ inosine $>\mathrm{AMP}>\mathrm{GMP}>$ guanosine $>$ adenosine> hypoxanthine. Among these metabolites, the high IMP production of $13.63 \pm 0.78 \mathrm{~g} / \mathrm{L}$ provided a sufficient precursor for the synthesis of various purine intermediates. Therefore, the universal chassis strain was successfully constructed to produce various purine intermediates under the guidance of in silico-guided engineering strategy, providing an effective and universal approach for metabolic engineering of the purine biosynthesis pathway.

\section{Discussion}


In this study, a rational-designed strategy based on the genome-scale metabolic network model succeeds in maximizing the biosynthesis of purine intermediates and biomass. With advantage over previous researches, the method fully understands the global regulation and metabolic flux to construct a general purine chassis strain for the first time and achieve the highest yield of inosine in de novo engineered bacteria. Based on the metabolic pathway, purine nucleosides are not the end products and can be converted into ribose and base by the salvage pathway, resulting in a decline of nucleoside accumulation [4]. In bacteria, the decomposition of purine nucleosides is mainly reversibly catalyzed by the purine-nucleoside phosphorylase PNP (encoding by deoD or pupG genes) (Fig. 3a). In previous studies, deoD and/or pupG were always knocked out to increase the accumulation of purine nucleosides $[13,14]$. However, the biomass was usually reduced dramatically in the engineered strains, which were difficultly for further optimization [12]. Consequently, the lack of understanding the global regulation and metabolic network resulting in poor cell performance and low production [17]. Therefore, it is necessary to block the conversion of nucleosides into other substances without affecting cell growth as much as possible. Based on the computational prediction, the Drm enzyme was found to be an essential backflow node of the purine synthesis pathway toward PPP. The study firstly proved that Drm deficiency could preferentially block the decomposition of nucleosides and considerably increase the production of purine nucleosides without an obvious impact on cell growth (Fig. 2). To validate the predicted target, the $d r m$ gene was inactivated in different ways since the $d r m$ and pup $G$ genes are located in the same operon in $B$. subtilis. Although the mRNA expression levels of the pupG gene were different in these $d r m$ inactivation strains, the inosine productions were all increased to $13.98-14.47 \mathrm{~g} / \mathrm{L}$. The results indicated that the $d r m$ gene played a major effect on blocking the inosine decomposition, which was further verified by the complementary experiment (Additional file 1: Figure S1). Therefore, this study firstly proved that loss of Drm function can effectively block the decomposition of purine nucleosides and further research is required to investigate the underlying mechanism.

The purine synthesis pathway is strictly regulated by complex regulatory mechanisms and coupled with amino acids, folic acids, and energy metabolism[28]. To effectively increase nucleoside synthesis, it is particularly important to release the complex regulations of purine operon (Fig. 3a). The pur operon (purEKBCQLFMNHD) encoding 11 enzymes is necessary for de novo synthesis of the precursor IMP of the purine intermediates in $B$. subtilis. However, transcription of the operon is dually regulated by a repressor protein encoded by the purR gene [29] and a riboswitch controlled by purine levels [16]. The regulatory sequences are located in the intergenic sequence upstream of the operon. Usually, the expression level of the purine operon was increased by deleting the purR gene, destroying the riboswitch structure, or optimizing the core sequence of the pur operon promoter region $[3,13,30]$. In this study, the promoter sequence of the purine operon was replaced with the constitutive promoters of different strengths (including $\mathrm{P}_{43}, \mathrm{P}_{\text {veg}}, \mathrm{P}_{c t o}$ and $\mathrm{P}_{g s i B}$ ) to eliminate the pur Box sequence and riboswitch structure of the PurR recognition site in the original promoter region, which can simply and effectively relieve the complex regulation. Combining the transcription level and translation initiation efficiency (Fig. 4a, b), the highest expression level of the purine operon is the W168 strain with $\mathrm{P}_{c t o}$ followed by $\mathrm{P}_{\text {veg }}$ or $\mathrm{P}_{g s i B^{\prime}}$ and the lowest is $\mathrm{P}_{43}$. However, $\mathrm{P}_{\text {veg }}$ reached the highest nucleosides accumulations in the W168 (Fig. 4c), 
possibly attributing to the existence of the post-transcriptional/translational modification in the regulation of the purine operon [31]. Consequently, inosine production was significantly increased to $16.86 \pm 0.78 \mathrm{~g} / \mathrm{L}$ in the inosine engineered strain by replacing $\mathrm{P}_{\text {pur }}$ with $\mathrm{P}_{\text {veg }}($ Fig. $4 \mathrm{~d})$.

Due to the complexity of the purine metabolic network, the metabolic flow of nucleosides might be difficult to be enhanced through traditional metabolic engineering [15]. Firstly, PPP, the carbon carrier pathway for purine nucleotide synthesis, is not the mainstream pathway in cells and the metabolic flow is relatively low [12]. Secondly, when the metabolites of PPP increase, they will return to the central carbon metabolism pathway (Fig. 5a). Based on this, GEM was used to predict an essential target transaldolase YwjH, which can reversibly catalyze Erythrose-4-phosphate (E4P) and Sedoheptulose-7-phosphate (S7P) in PPP to form Fructose-6-phosphate (F6P) and pyruvate in EMP. According to the analysis of the metabolic network, YwjH deficiency blocks the PPP flow to the EMP. Additionally, overexpression of transaldolase has been reported to affect the synthesis of Ribose-5-phosphate (R5P) and increasing histidine accumulation [32]. On the opposite, the deficiency of transaldolase could weaken the competitive pathways and promote the synthesis of ribose-5-P. The metabolic flow of PRPP synthesis was thereof enhanced, contributing to the accumulation of purine nucleosides. As a result, the $y w j H$ knockout significantly increased the inosine production to $22.01 \pm 1.18 \mathrm{~g} / \mathrm{L}$ (Fig. 5a).

The performance of the inosine engineered strain was further optimized to balance cell growth and inosine production by constructing a dynamic switch. Glucose is phosphorylated by the glucokinase GlcK to synthesize Glucose-6- phosphate (G6P), which can be catalyzed either by glucose-6-P-1-dehydrogenase Zwf to form Glucono-1,5-L-6P of PPP or by glucose-6-P isomerase Pgi to form F6P of EMP (Fig. 3a). To all our knowledge, the protein scaffolding strategy is designed by the principle of interaction between scaffold proteins and their ligands to combine multiple targets on the metabolic network into a linear pathway and rebuild a multi-enzyme-catalyzed biological reaction system in the cell [33]. The technology has been successfully applied to the accumulation of target products such as mevalonic acid, saccharic acid, alkanes, and butanediol [34-37]. To dynamically control the metabolic flow of PPP and EMP, the scaffold proteins GBD, SH3, and PDZ were separately used to linearly assemble $g / c K, z w f$, and $y k g B$ expressed by the inducible promoter $\mathrm{P}_{\text {grac }}$ (Fig. $5 \mathrm{~b}$ ). The inosine production did not change under the control of the inducer except for the reduced biomass by the addition of the inducer, suggesting the inefficient effect on dynamical control of the metabolic flow. Then, the pgi expression level controlled by the inducible promoter $\mathrm{P}_{x y}$ was used to establish an efficient dynamic switch (Fig. 5c). Notably, the inosine production greatly decreased by overexpressing the pgi gene under the induction condition but increased in the absence of an inducer. Meanwhile, the cell growth greatly improved by adding inducer but decreased by removing the inducer, suggesting a potential switch to dynamically regulate the biomass and product. The expression intensity of the pgi gene was further adapted as an effective metabolic switch to balance the product and biomass, achieving the inosine production of $25.81 \pm 1.23$ $\mathrm{g} / \mathrm{L}$ by shake-flask cultivation (Fig. $5 \mathrm{~d}$ and Additional file 1: Figure S7). However, some other factors such as the expression intensity of the multi-enzyme catalytic system and the ratio of each enzyme's expression level might also affect the inosine synthesis. It is necessary to finely control the expression 
levels of the key enzymes in the metabolic pathway to achieve the joint and cooperative expression of multiple genes in further study [38].

In conclusion, GEM was used to predict metabolic targets for optimization of the purine nucleoside synthesis. Under the guidance of in silico prediction, the production of purine nucleosides was remarkably improved by optimizing the targets $d r m, \mathrm{P}_{\text {pun }} y w j H, z w f$, and $p g i$ to block the nucleosides degradation, strengthen PPP, and weaken EMP. Under the two-stage fermentation by a pgi-based metabolic switch, the engineered strain produced up to $25.81 \pm 1.23 \mathrm{~g} / \mathrm{L}$ of inosine without affecting cell growth by the shakeflask cultivation, suggesting the highest inosine production in de novo engineered bacteria to data. Finally, the in silico-guided metabolic engineering strategy was successfully used to generate the universal chassis bacterium capable of producing various purine intermediates, such as inosine, adenosine, guanosine, IMP, and GMP. Therefore, the rationally engineering strategy based on GEM was successfully used to resolve a competitive relationship between cell growth and nucleoside synthesis, suggesting potential applications for a wide range of biotechnology products.

\section{Abbreviations}

IMP: inosine monophosphate; EMP: Glycolysis pathway/Embden-Meyerhof-Parnas Pathway; PPP: Pentose Phosphate Pathway; PRPP: phosphoribosyl pyrophosphate; AMP: adenosine 5'-monophosphate ; XMP: xanthosine 5'-monophosphate; GMP: guanosine 5'-monophosphate; GEM: genome-scale metabolic network models; FBA: flux balance analysis; GDLS: genetic design through local search; ROOM: minimum switch adjustment algorithm; PNP: purine nucleoside phosphorylases; ORF: opening reading frame; RBS: ribosome binding site; $B$. subtilis: Bacillus subtilis; HPLC: high-performance liquid chromatography; $\mathrm{OD}_{600}$ : optical density at wavelength $(\lambda) 600 \mathrm{~nm}$; WT: wild type; BS: Bacillus subtilis; SOE: splice overlap extension; SD: standard deviations; qRT-PCR: real-time quantitative reverse transcription PCR.

\section{Declarations}

\section{Authors' Contributions}

TW and AD conceived and designed the study. AD, QQ, and QS performed the experiments and all data analysis with assistance from $Z C, Y Z$, and JW. AD wrote the manuscript with the revision from TW and SW. All of the authors have approved the final paper.

\section{Author details}

${ }^{1}$ CAS Key Laboratory of Pathogenic Microbiology and Immunology, Institute of Microbiology, Chinese Academy of Sciences, Beijing 100101, China. ${ }^{2}$ College of Life Sciences, University of Chinese Academy of

Sciences, Beijing 100049, China. ${ }^{3}$ Savaid medical school, University of Chinese Academy of Sciences, Beijing, 100049 , China. ${ }^{4}$ China Innovation Academy for Green Manufacture, Chinese Academy of Sciences, Beijing, 100049, China 
Acknowledgments

We are particularly grateful to the Pathogenic Microbiology and Immunology Public Technology Service Center for its support.

\section{Competing interests}

The authors declare that they have no competing interests.

\section{Availability of supporting data}

Data supporting the results of the article are included within this manuscript.

\section{Consent for publication}

All authors have approved the manuscript for publication.

\section{Funding}

This work was supported by grants from the Strategic Priority Research Program of the Chinese Academy of Sciences (XDA17010503), the National Natural Science Foundation of China (31570083 and 31170103), and the Innovation Academy for Green Manufacture, Chinese Academy of Sciences (IAGM2019-A02).

\section{References}

1. Kappock TJ, Ealick SE, Stubbe J: Modular evolution of the purine biosynthetic pathway. Curr Opin Chem Biol. 2000, 4(5):567-572.

2. Jimenez A, Santos MA, Pompejus M, Revuelta JL: Metabolic engineering of the purine pathway for riboflavin production in Ashbya gossypii. Appl Environ Microbiol. 2005, 71(10):5743-5751.

3. Liu M, Fu YX, Gao WJ, Xian M, Zhao G: Highly efficient biosynthesis of hypoxanthine in Escherichia coli and transcriptome-based analysis of the purine metabolism. ACS Synth Biol. 2020, 9(3):525535.

4. Shi T, Wang Y, Wang Z, Wang G, Liu D, Fu J, Chen T, Zhao X: Deregulation of purine pathway in Bacillus subtilis and its use in riboflavin biosynthesis. Microb Cell Fact. 2014, 13(1):101.

5. Smith JL, Zaluzec EJ, Wery JP, Niu L, Switzer RL, Zalkin H, Satow Y: Structure of the allosteric regulatory enzyme of purine biosynthesis. Science. 1994, 264(5164):1427-1433.

6. Li B, Yan ZY, Liu XN, Zhou J, Wu XY, Wei P, Jia HH, Yong XY: Increased fermentative adenosine production by gene-targeted Bacillus subtilis mutation. J Biotechnol. 2019, 298:1-4.

7. Zhang CL, Du SS, Liu Y, Xie XX, Xu QY, Chen N: Strategy for enhancing adenosine production under the guidance of transcriptional and metabolite pool analysis. Biotechnol Lett. 2015, 37(7):13611369. 
8. Zakataeva NP, Romanenkov DV, Skripnikova VS, Vitushkina MV, Livshits VA, Kivero AD, Novikova AE: Wild-type and feedback-resistant phosphoribosyl pyrophosphate synthetases from Bacillus amyloliquefaciens: purification, characterization, and application to increase purine nucleoside production. Appl Microbiol Biotechnol. 2012, 93(5):2023-2033.

9. Sheremet AS, Gronskiy SV, Akhmadyshin RA, Novikova AE, Livshits VA, Shakulov RS, Zakataeva NP: Enhancement of extracellular purine nucleoside accumulation by Bacillus strains through genetic modifications of genes involved in nucleoside export. J Ind Microbiol Biotechnol. 2011, 38(1):65-70.

10. Wu HY, Li YJ, Ma Q, Li Q, Jia ZF, Yang B, Xu QY, Fan XG, Zhang CL, Chen N et al: Metabolic engineering of Escherichia coli for high-yield uridine production. Metab Eng. 2018, 49:248-256.

11. Sauer U, Cameron DC, Bailey JE: Metabolic capacity of Bacillus subtilis for the production of purine nucleosides, riboflavin, and folic acid. Biotechnol Bioeng. 1998, 59(2):227-238.

12. Li HJ, Zhang GQ, Deng AH, Chen N, Wen TY: De novo engineering and metabolic flux analysis of inosine biosynthesis in Bacillus subtilis. Biotechnol Lett. 2011, 33(8):1575-1580.

13. Asahara T, Mori Y, Zakataeva NP, Livshits VA, Yoshida K, Matsuno K: Accumulation of gene-targeted Bacillus subtilis mutations that enhance fermentative inosine production. Appl Microbiol Biotechnol. 2010, 87(6):2195-2207.

14. Shimaoka M, Takenaka Y, Kurahashi O, Kawasaki H, Matsui H: Effect of amplification of desensitized purF and prs on inosine accumulation in Escherichia coli. J Biosci Bioeng. 2007, 103(3):255-261.

15. Switzer RL: Discoveries in bacterial nucleotide metabolism. J Biol Chem. 2009, 284(11):6585-6594.

16. Mandal M, Boese B, Barrick JE, Winkler WC, Breaker RR: Riboswitches control fundamental biochemical pathways in Bacillus subtilis and other bacteria. Cell. 2003, 113(5):577-586.

17. Ramazzina I, Costa R, Cendron L, Berni R, Peracchi A, Zanotti G, Percudani R: An aminotransferase branch point connects purine catabolism to amino acid recycling. Nat Chem Biol. 2010, 6(11):801806.

18. Pabinger S, Rader R, Agren R, Nielsen J, Trajanoski Z: MEMOSys: Bioinformatics platform for genome-scale metabolic models. BMC Syst Biol. 2011, 5:9.

19. Monk JM, Lloyd CJ, Brunk E, Mih N, Sastry A, King Z, Takeuchi R, Nomura W, Zhang Z, Mori H et al: iML1515, a knowledgebase that computes Escherichia coli traits. Nat Biotechnol. 2017, 35(10):904908.

20. Kim B, Kim WJ, Kim DI, Lee SY: Applications of genome-scale metabolic network model in metabolic engineering. J Ind Microbiol Biotechnol. 2015, 42(3):339-348.

21. Oh YK, Palsson BO, Park SM, Schilling CH, Mahadevan R: Genome-scale reconstruction of metabolic network in Bacillus subtilis based on high-throughput phenotyping and gene essentiality data. J Biol Chem. 2007, 282(39):28791-28799.

22. Zhang Y, Cai JY, Shang XL, Wang B, Liu SW, Chai X, Tan TW, Zhang Y, Wen TY: A new genome-scale metabolic model of Corynebacterium glutamicum and its application. Biotechnol Biofuels. 2017, 10:16. 
23. Duarte NC, Herrgard MJ, Palsson BO: Reconstruction and validation of Saccharomyces cerevisiae iND750, a fully compartmentalized genome-scale metabolic model. Genome Res. 2004, 14(7):12981309.

24. Tanaka K, Henry CS, Zinner JF, Jolivet E, Cohoon MP, Xia FF, Bidnenko V, Ehrlich SD, Stevens RL, Noirot P: Building the repertoire of dispensable chromosome regions in Bacillus subtilis entails major refinement of cognate large-scale metabolic model. Nucleic Acids Res. 2013, 41(1):687-699.

25. Lun DS, Rockwell G, Guido NJ, Baym M, Kelner JA, Berger B, Galagan JE, Church GM: Large-scale identification of genetic design strategies using local search. Mol Syst Biol. 2009, 5:8.

26. Shlomi T, Berkman O, Ruppin E: Regulatory on/off minimization of metabolic flux changes after genetic perturbations. Proc Natl Acad Sci USA. 2005, 102(21):7695-7700.

27. Salis HM, Mirsky EA, Voigt CA: Automated design of synthetic ribosome binding sites to control protein expression. Nat Biotechnol. 2009, 27(10):946-952.

28. Rebora K, Laloo B, Daignan-Fornier B: Revisiting purine-histidine cross-pathway regulation in Saccharomyces cerevisiae: a central role for a small molecule. Genetics. 2005, 170(1):61-70.

29. Weng M, Nagy PL, Zalkin H: Identification of the Bacillus subtilis puroperon repressor. Proc Natl Acad Sci USA. 1995, 92:7455-7459.

30. Matsui H, Kawasaki H, Shimaoka M, Kurahashi O: Investigation of various genotype characteristics for inosine accumulation in Escherichia coli W3110. Biosci Biotechnol Biochem. 2001, 65(3):570578.

31. Ebbole DJ, Zalkin H: Bacillus subtilis pur operon expression and regulation. J Bacteriol. 1989, 171(4):2136-2141.

32. Anasontzis GE, Kourtoglou E, Mamma D, Villas-Boas SG, Hatzinikolaou DG, Christakopoulos P: Constitutive homologous expression of phosphoglucomutase and transaldolase increases the metabolic flux of Fusarium oxysporum. Microb Cell Fact. 2014, 13:12.

33. Bashor CJ, Helman NC, Yan SD, Lim WA: Using engineered scaffold interactions to reshape MAP kinase pathway signaling dynamics. Science. 2008, 319(5869):1539-1543.

34. Dueber JE, Wu GC, Malmirchegini GR, Moon TS, Petzold CJ, Ullal AV, Prather KLJ, Keasling JD: Synthetic protein scaffolds provide modular control over metabolic flux. Nat Biotechnol. 2009, 27(8):753-759.

35. Rahman Z, Sung BH, Yi J-Y, Bui LM, Lee JH, Kim SC: Enhanced production of n-alkanes in Escherichia coli by spatial organization of biosynthetic pathway enzymes. J Biotechnol. 2014, 192:187-191.

36. Kim S, Hahn JS: Synthetic scaffold based on a cohesin-dockerin interaction for improved production of 2,3-butanediol in Saccharomyces cerevisiae. J Biotechnol. 2014, 192:192-196.

37. Liu YF, Zhu YQ, Ma WL, Shin HD, Li JH, Liu L, Du GC, Chen J: Spatial modulation of key pathway enzymes by DNA-guided scaffold system and respiration chain engineering for improved Nacetylglucosamine production by Bacillus subtilis. Metab Eng. 2014, 24:61-69. 
38. Khalil AS, Collins JJ: Synthetic biology: applications come of age. Nat Rev Genet. 2010, 11(5):367379 .

39. Zhang GQ, Wang WZ, Deng AH, Sun ZP, Zhang Y, Liang Y, Che YS, Wen TY: A Mimicking-of-DNAMethylation-Patterns pipeline for overcoming the restriction barrier of bacteria. PLoS Genet. 2012, 8(9):e1002987.

40. Wu J, Deng A, Sun Q, Bai H, Sun Z, Shang X, Zhang Y, Liu Q, Liang Y, Liu S et al: Bacterial genome editing via a designed toxin-antitoxin cassette. ACS Synth Biol. 2018, 7(3):822-831.

41. Deng A, Sun Z, Wang T, Cui D, Li L, Liu S, Huang F, Wen T: Simultaneous multiplex genome engineering via accelerated natural transformation in Bacillus subtilis. Front Microbiol. 2021, 12(714449).

42. Hao T, Han B, Ma H, Fu J, Wang H, Wang Z, Tang B, Chen T, Zhao X: In silico metabolic engineering of Bacillus subtilis for improved production of riboflavin, Egl-237, (R,R)-2,3-butanediol and isobutanol. Molecular Biosystems. 2013, 9(8):2034-2044.

43. Saifuddin M, Bhatnagar JM, Segre D, Finzi AC: Microbial carbon use efficiency predicted from genome-scale metabolic models. Nat Commun. 2019, 10:10.

44. Kleessen S, Nikoloski Z: Dynamic regulatory on/off minimization for biological systems under internal temporal perturbations. BMC Syst Biol. 2012, 6:13.

45. Schmittgen, D T, Livak, J K: Analyzing real-time PCR data by the comparative CT method. Nat Protoc. 2008, 3(6):1101-1108.

\section{Figures}




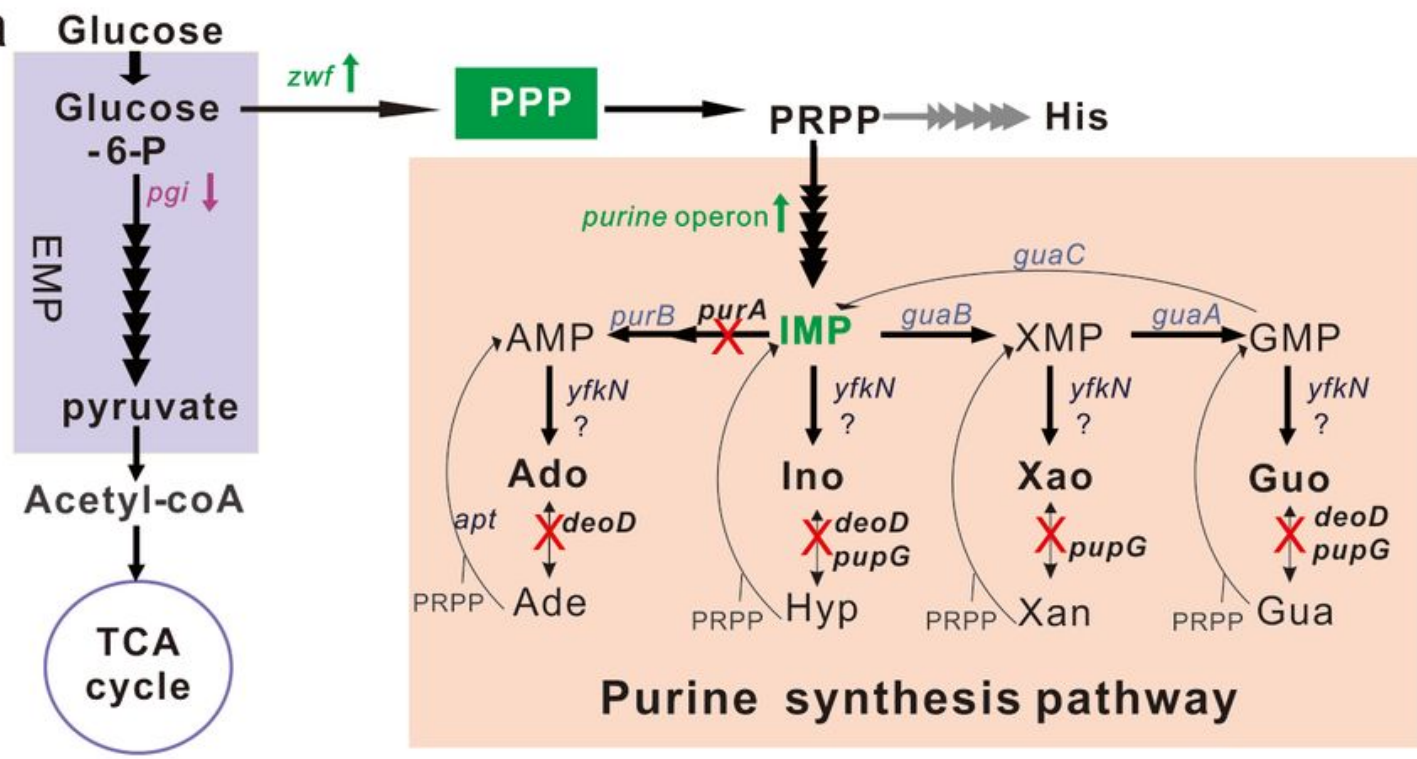

b
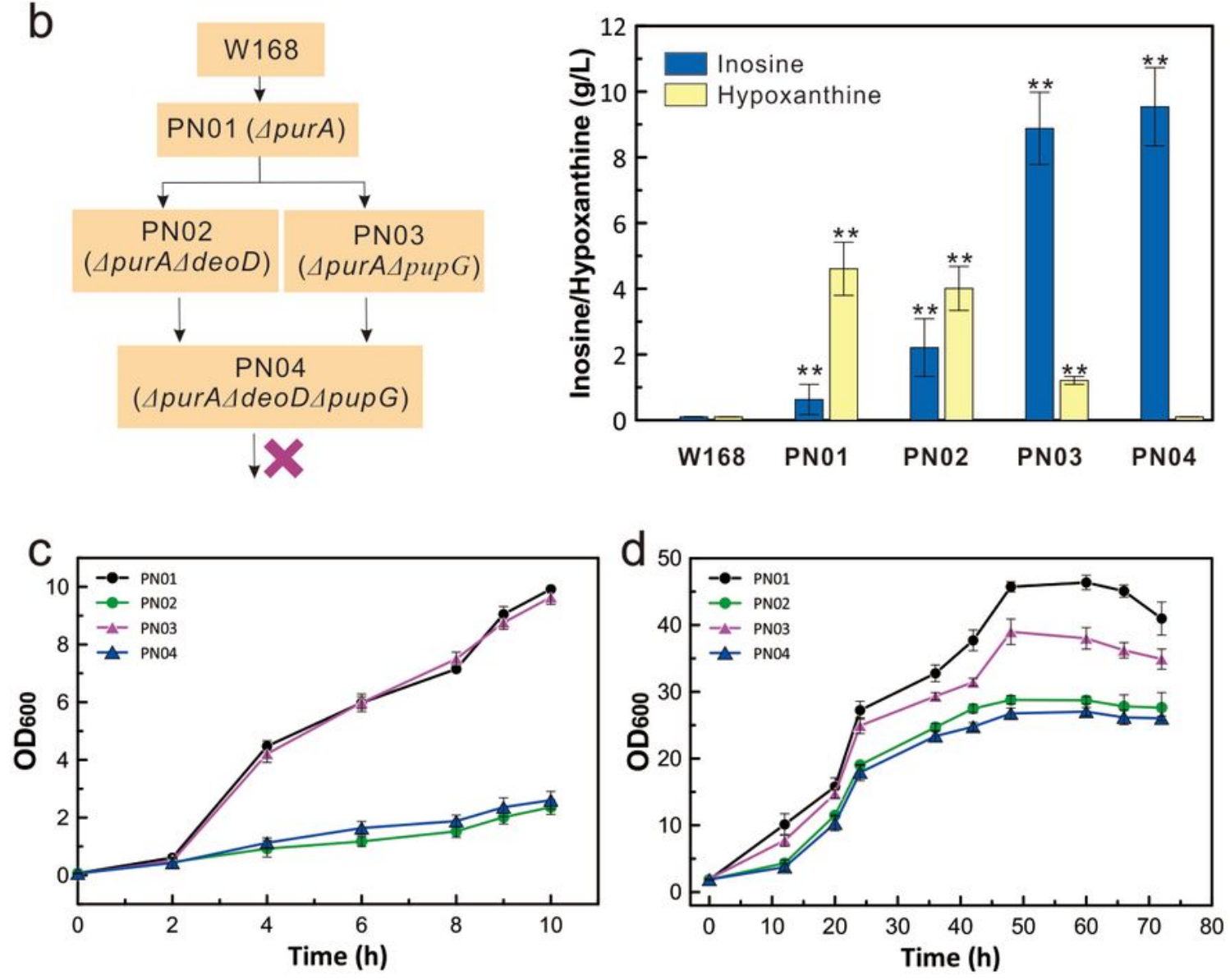

\section{Figure 1}

Traditional engineering of Bacillus subtilis for de novo synthesis of purine nucleosides. (a) Biosynthetic pathway of purine nucleosides in B. subtilis. prs, encoding PRPP synthetase; purEKBCSQLFMNHD (pur operon), encoding enzymes required to synthesize IMP from PRPP; Ppur, purine operon promoter; purA, encoding adenylosuccinate synthase; purB, encoding adenylosuccinate lyase; guaB, encoding IMP dehydrogenase; guaA, encoding GMP synthetase; guaC, encoding GMP reductase; deoD, encoding purine 
nucleoside phosphorylase (PNP); pupG, encoding PNP; apt, encoding adenine phosphoribosyltransferase; hpt, encoding hypoxanthine-guanine phosphoribosyltransferase; xpt, encoding xanthine phosphoribosyltransferase; Ado, adenosine; Ino, inosine; Xao, xanthosine; Guo, guanosine; Ade, adenine; Hyp, hypoxanthine; Xan, xanthine; Gua, guanine. (b) Construction of engineered strains for de novo synthesis of inosine by traditional metabolic engineering. (c) The growth curve in seed cultivation. (d) Growth curve by shake-flask cultivation. Data shown are mean values from three biological replicates and a value of $P$ less than 0.05 is regarded to be a significant difference with the W168 strain using the T-test $(* \star, P<0.01)$. 


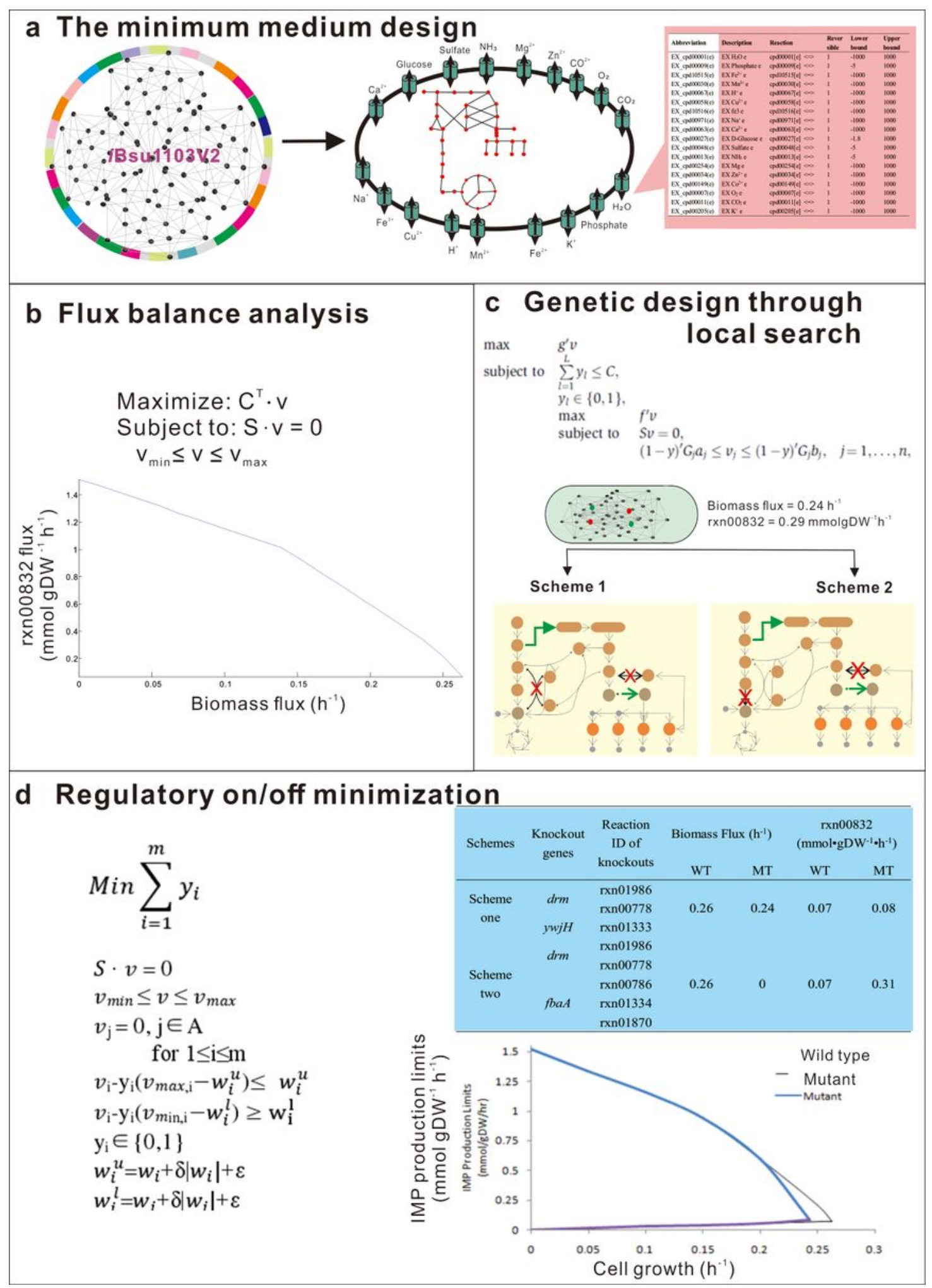

Figure 2

Applications of the genome-scale metabolic network models (GEM) to predict the optimal targets. (a) The minimum medium design through GEM. The minimum medium was designed in the $B$. subtilis model for the simulation calculation. (b) Analysis of IMP flux and Biomass flux by FBA. (c) Prediction of the potential targets by GDLS. (d) Flow disturbance analysis of the mutants by ROOM. Maximal and minimal IMP production rates were predicted at different growth rates. 

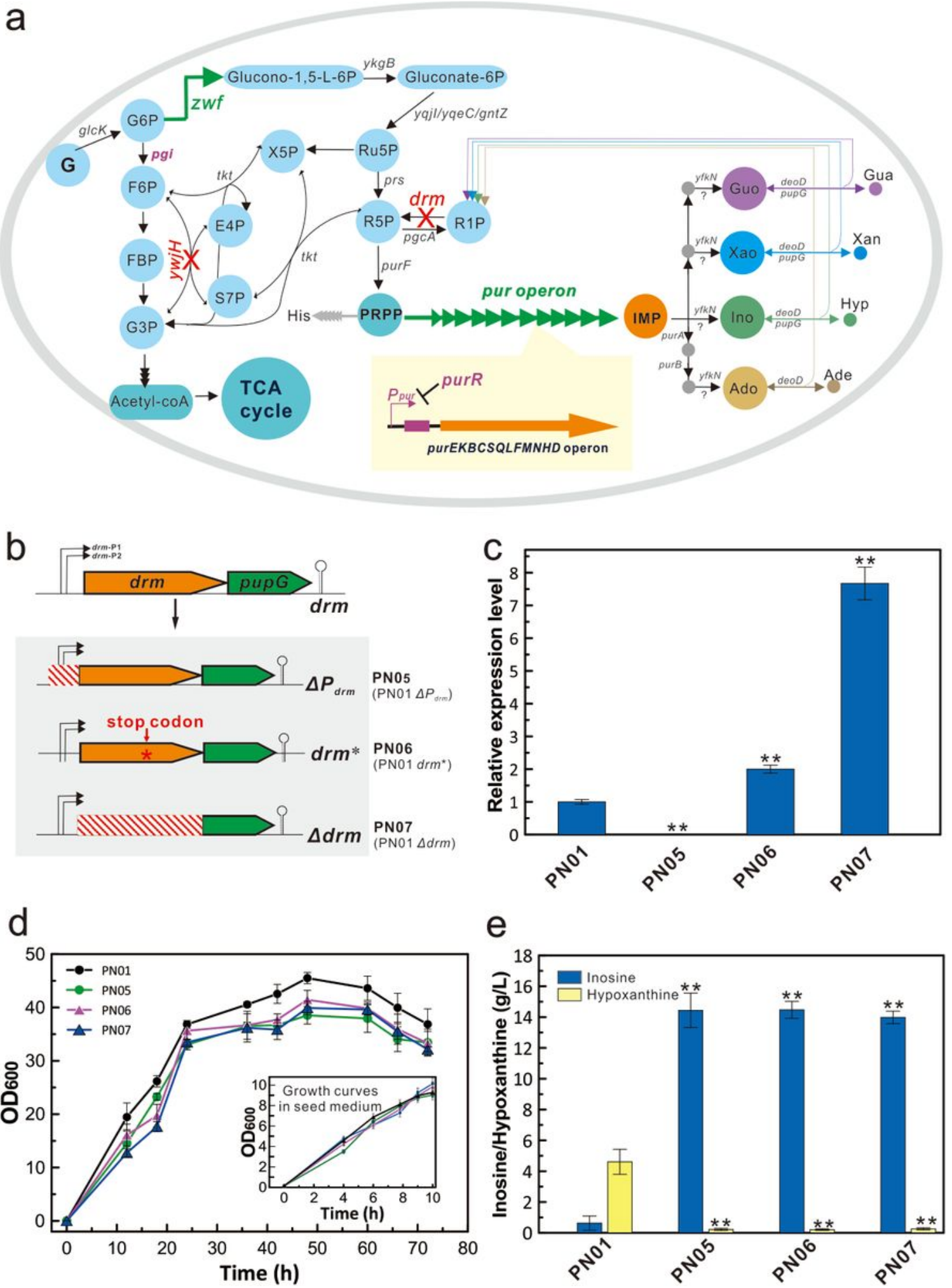

\section{Figure 3}

The performance of the Drm-inactivated strains. (a) The engineering strategy proposed by GSM for de novo synthesis of purine nucleosides in B. subtilis. Genes/reactions in red indicate deletion and genes/reactions in green indicate overexpression. glcK, encoding glucokinase; zwf, encoding glucose-6phosphate 1-dehydrogenase; $\mathrm{ykgB}$, encoding 6-phosphogluconolactonase ; pgi, encoding glucose-6phosphate isomerase; ywjH, encoding transaldolase; tkt, encoding transketolase; prs, encoding ribose- 
phosphate pyrophosphokinase; purF, encoding amidophosphoribosyltransferase; drm, encoding pentose phosphate mutase; pgcA, encoding phosphoglucomutase; purR, Pur operon repressor. (b) The schematic diagram for the modifying strategies of drm inactivation. The engineered strains PN05, PN06, and PN07 were constructed by nonsense point mutation of the drm gene, deletion of the promoter Pdrm, and knockout of the open reading frame, respectively. (c) The relative mRNA expression level of pupG gene in the engineering strains. The relative transcriptional levels were analyzed by quantitative real-time PCR using PN01 as the control. (d) The cell growth during shake-flask cultivation. The inset shows the growth curves in the seed medium. (e) The effect of the drm deficiency on the production of inosine and hypoxanthine. All error bars indicate $\pm S D, n=3$. A value of $P$ less than 0.05 was regarded to be a significant difference with the control strain PN01 using the T-test ( $* *, P<0.01)$.

a
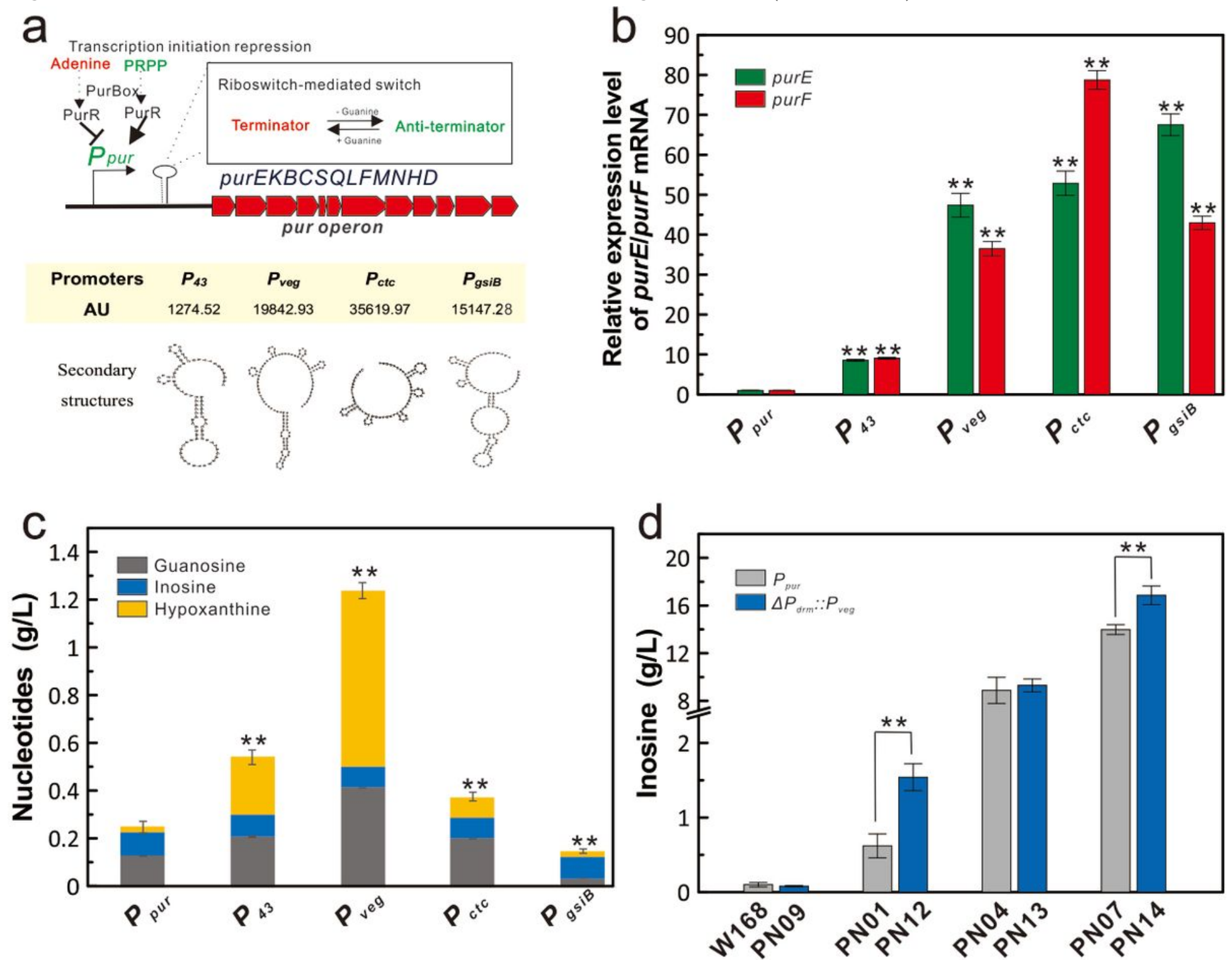

Figure 4

The replacement of promoter Ppur to release feedback regulation of key enzymes in purine synthesis. (a) Different strength of promoters selected to release the transcription initiation repression on the pur 
operon. The translation initial efficiencies of promoters are predicted by RBS calculator v1.1 [27]. The secondary structures formed between 5'-UTR and purE are predicted by DNAMAN. (b) The relative mRNA expression level of purE and purF genes under different promoters. The relative transcriptional levels are analyzed by quantitative real-time PCR using Ppur as the control. (c) The effect of promoter replacement on the accumulation of purine intermediates in the W168 strain. (d) The effect of the promoter replacement on the accumulation of inosine in the engineered strains. The promoter Pveg was used to replace Ppur in the strains W168, PN01, PN04, and PN07, separately producing strains PN08 (W168 Ppur::Pveg), PN12 (W168 $\Delta$ purA Ppur::Pveg), PN13 (W168 $\Delta$ purA $\triangle$ pupg Ppur::Pveg), and PN14 (W168 $\triangle$ purA $\Delta$ drm Ppur::Pveg). All error bars indicate $\pm S D, n=3$. A value of $P$ less than 0.05 was regarded to be a significant difference from the original promoter Ppur $(* \star, P<0.01)$. 

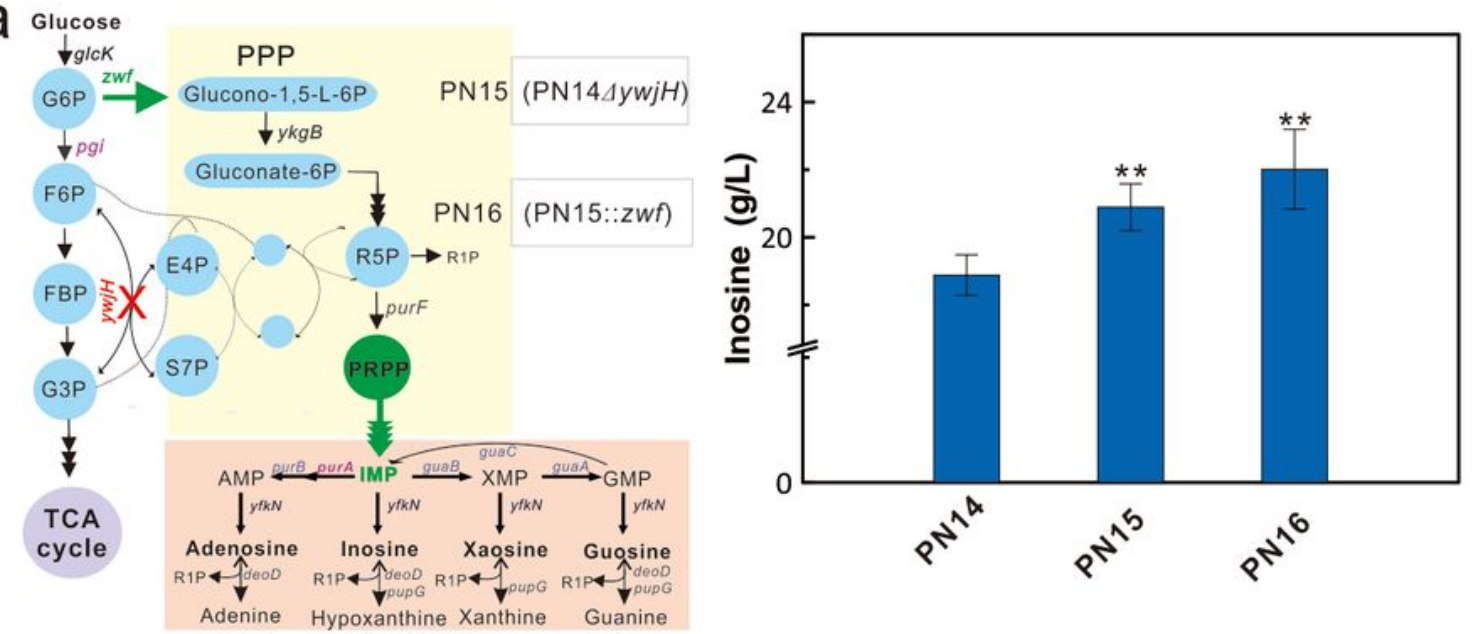

b

Purine nucletides synthesis pathway
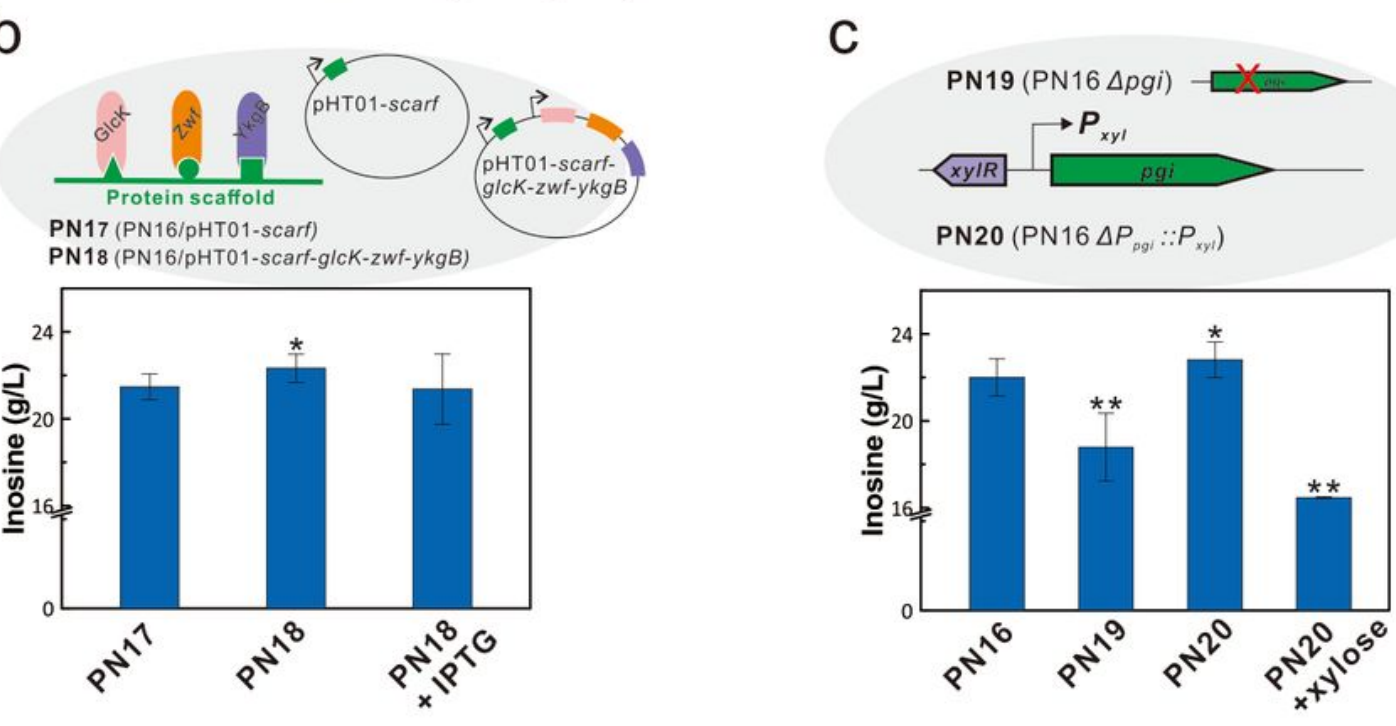

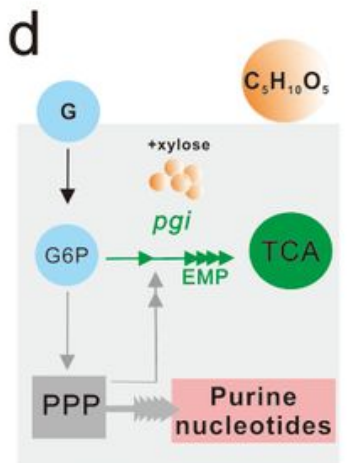

Cell growth

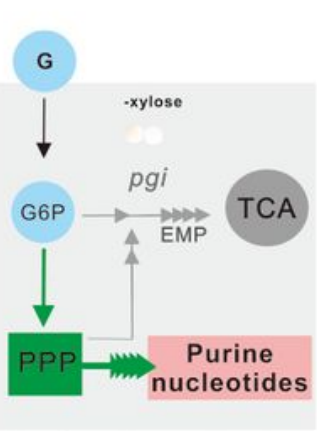

Nucleotide synthesis

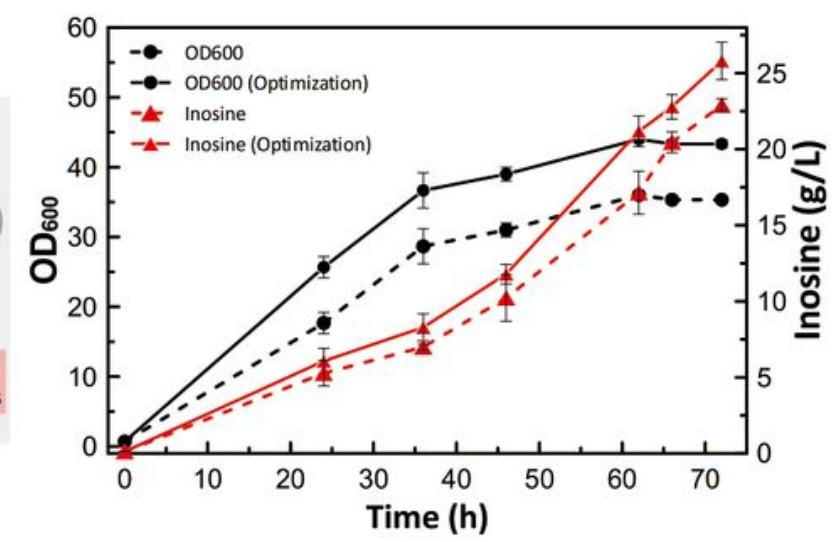

\section{Figure 5}

Overproduction of inosine by enhancing the metabolic flow of PPP. (a) Inosine productions of the engineered strains by knocking out the ywjH gene and overexpressing the zwf gene. (b) The effect of linearly assembling proteins GlcK, Zwf, and YkgB on the inosine synthesis. The protein scaffolds GBD, $\mathrm{SH} 3$, and PDZ were separately used to linearly assemble proteins GlcK, Zwf, and YkgB. (c) The effect of the pgi expression level on the inosine synthesis. Conditional expression of the pgi gene under the control 
of the Pxyl promoter was used to balance the inosine production and cell growth. (d) Two-stage fermentation in the inosine engineered strain by the pgi-based metabolic switch. All error bars indicate \pm $S D, n=3$. A value of $P$ less than 0.05 was regarded to be a significant difference with the W168 strain using the T-test $(*, P<0.05 ; * *, P<0.01)$.

a

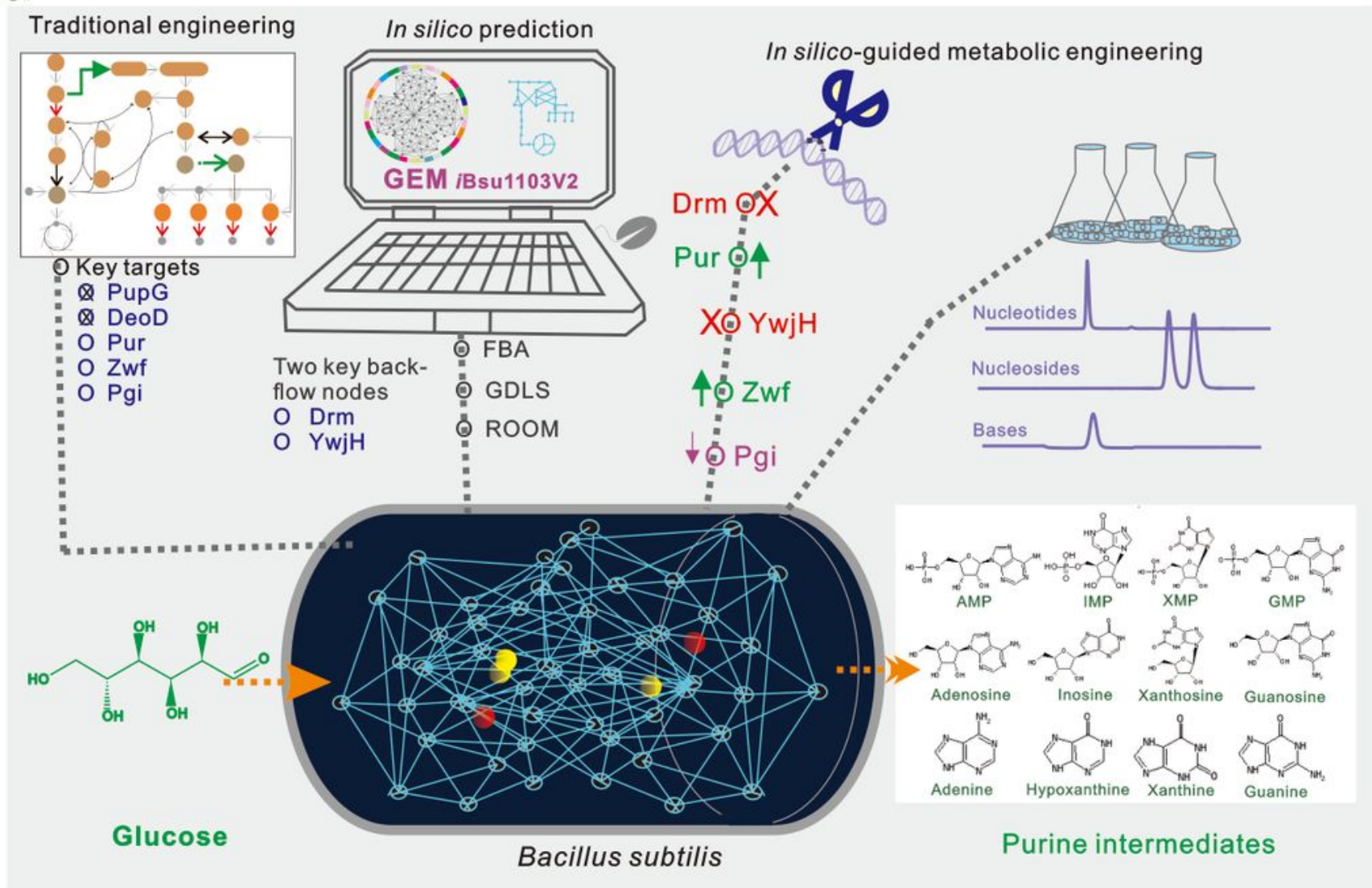

b

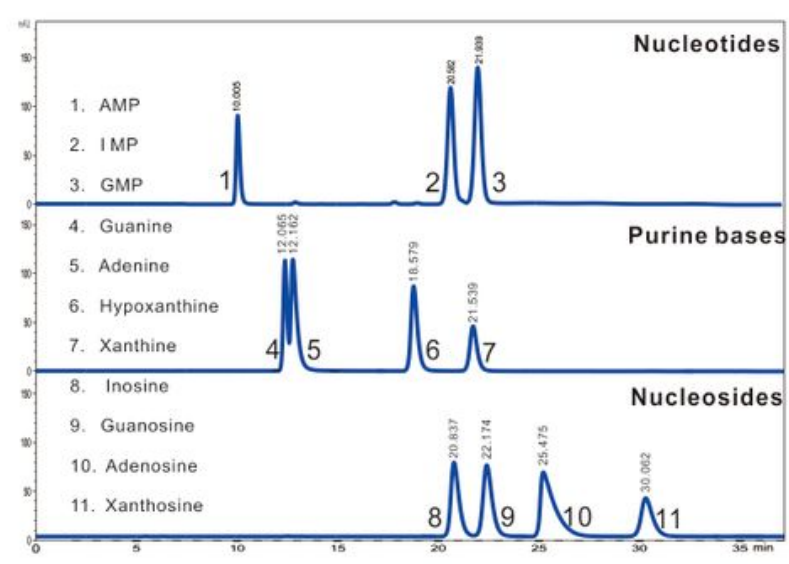

C

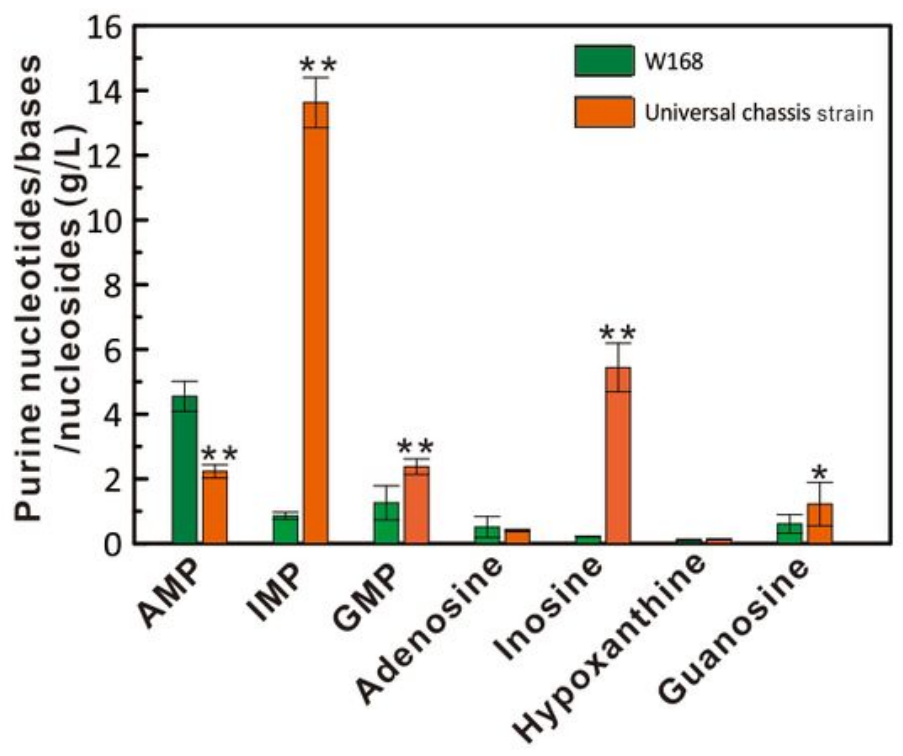

Figure 6 
Construction of the general purine chassis bacterium by in silico-guided metabolic engineering strategy. (a) Schematic diagram for in silico-guided metabolic engineering strategy. (b) The retention times of purine nucleosides, bases, and nucleosides by HPLC. (c) Determination of purine nucleosides metabolites synthesized in the general purine chassis strain. All error bars indicate $\pm S D, n=3$. A value of $P$ less than 0.05 was regarded to be a significant difference with the W168 strain using the T-test $\left({ }^{*}, \mathrm{P}<0.05 ; * \star, P<\right.$ $0.01)$.

\section{Supplementary Files}

This is a list of supplementary files associated with this preprint. Click to download.

- Supportinformation10.doc 